Original
article

Institute of Tropical Medicine, Antwerp,

Belgium

L Van Damme

K Depraetere

$V$ Vandersmissen

E Van Dyck

M Laga

Imperial College

School of Medicine,

St Mary's Hospital,

London

A Wright

I Rosenstein

L Poulter

J Weber

V Kitchen

Procept Inc,

Cambridge, MA, USA

M McKinlay

A Profy

Correspondence to: Dr Lut Van Damme, STD/HIV Research and Intervention Unit, Institute of Tropical Medicine, Nationalestraat 155, 2000 Antwerp, Belgium email: lvandamme@itg.be

Accepted for publication 20 January 2000

\title{
A phase I study of a novel potential intravaginal microbicide, PRO 2000, in healthy sexually inactive women
}

\author{
Lut Van Damme, Ann Wright, Katrien Depraetere, Isobel Rosenstein, \\ Veerle Vandersmissen, Len Poulter, Margo McKinlay, Eddy Van Dyck, Jonathan Weber, \\ Al Profy, Marie Laga,Val Kitchen
}

Background: Although the male condom provides a reliable means of preventing HIV transmis-
sion, a broader choice of methods is required particularly in circumstances where the negotiation
of condom use is difficult. Development of new products that may be effective as topical vaginal
microbicides is the focus of a great deal of research activity currently. The novel agent PRO 2000,
a naphthalene sulphonate derivative with in vitro activity against HIV and other sexually
transmissible pathogens, is one such compound. We have studied the local and systemic safety
and tolerance of a vaginal gel formulation of this agent at two concentrations $(0.5 \%$ and $4 \%$ ) over
a 2 week period of daily exposure in two cohorts of healthy sexually abstinent women (one in
London, UK, and the other in Antwerp, Belgium). Methods: This was a randomised, placebo controlled, double blind, three arm clinical trial conducted on two sites. Macroscopic evidence of genital epithelial changes was sought using colposcopy and evidence of microscopic inflammation was acquired using high vaginal biopsy from predetermined sites (UK cohort only). Blood levels of PRO 2000 were measured and laboratory safety tests, including coagulation screens, were performed. The impact on vaginal ecology was also assessed.

Results: 73 women were enrolled across both sites (36 UK, 37 Belgium); 24, 24, 25 in the 4\%, $0.5 \%$, and placebo groups respectively. Of these, 70 completed 2 weeks' exposure to the study gel. Three (all in the $4 \%$ group) withdrew owing to adverse events which were possibly or probably gel related. Cervicovaginal abrasion was seen colposcopically in three subjects after 14 days of gel use (two in the $4 \%$ group and one in the placebo group). Genital ulceration was not seen during gel use in any of the subjects who completed the study. Histological evaluation of vaginal biopsy samples (36 women only) showed evidence of increased inflammatory signs in one participant of the $4.0 \%$ group. One volunteer in the placebo group had moderate inflammation at screening and at follow up. Severe inflammation was not seen among any of the subjects tested. Plasma levels of PRO 2000 and laboratory safety tests showed no evidence of systemic absorption. No impact was seen on normal vaginal ecology in the UK cohort where samples were taken 12 hours after the last gel application.

Conclusion: In this phase I study PRO 2000 gel was found to be generally well tolerated with promising local and systemic safety profiles. The $0.5 \%$ gel was better tolerated than the $4 \%$ gel as fewer genital epithelial adverse events were seen in the former. Phase II studies are about to begin in sexually active women.

(Sex Transm Inf 2000;76:126-130)

Keywords: microbicide; PRO 2000; women; toxicity

\section{Introduction}

The development of effective microbicides for HIV prevention is needed to broaden the range of safer sex choices and to empower women in this choice. ${ }^{12}$ So far, most microbicide research in humans has been largely focused on nonoxynol-9 (N-9), a non-ionic surfactant with potent anti-HIV activity in vitro. ${ }^{3}{ }^{4}$ However, a recently published randomised, triple blind placebo controlled trial showed that a vaginal contraceptive film containing $70 \mathrm{mg}$ of N-9 had no impact on the transmission of HIV, Neisseria gonorrhoeae, or Chlamydia trachomatis. ${ }^{5}$ At the present time the cause of this lack of effectiveness is unclear but it may be explained in part by the low therapeutic index of N-9 which could lead to epithelial disruption and/or inflammation thereby enhancing HIV transmission risk. ${ }^{6}$ Some investigators have found that the consistent use of N-9 may deplete lactobacilli and result in colonisation with abnormal bacterial species (mainly coliforms). ${ }^{7}$

The disappointing experiences with N-9 have led to an increased interest in the development of novel compounds with more specific anti-HIV activity. ${ }^{89}$ Such agents should preserve genital epithelial integrity and a normal vaginal flora, both of which are thought to mitigate HIV acquisition. ${ }^{10} 11$

PRO 2000 is a synthetic naphthalene sulphonate polymer that appears to be well suited for use as a vaginal microbicide. ${ }^{12}$ The compound has been shown to be active in vitro against HIV-1, HIV-2, herpes simplex viruses (HSV-1 and -2), human cytomegalovirus, and Chlamydia trachomatis. ${ }^{13}$ Furthermore, an aqueous vaginal gel formulation (PRO 2000 
gel) was shown to protect mice from genital HSV-2 infection. ${ }^{14}$ Gels containing up to $4 \%$ PRO 2000 were well tolerated in rabbit models for vaginal and penile irritation. ${ }^{12}{ }^{13}$ PRO 2000 gel is straightforward to manufacture, highly stable, and compatible with latex condoms. ${ }^{15}$

\section{Methods}

STUDY DESIGN

The study was approved by the institutional ethics committees at both sites. At each site 36 healthy female volunteers were randomised in a double blind manner. Each participant received $2 \mathrm{ml}$ doses of one of the following intravaginal preparations: $4 \%$ PRO 2000 gel $(80 \mathrm{mg}$ total dose), $0.5 \%$ PRO 2000 gel (10 mg total dose), or a matched placebo gel. The gel base contained a synthetic carbomer and an aqueous lactic acid/triethanolammonium lactate buffer ( $\mathrm{pH} 4.5$ ). The placebo comparator contained only these base components. Gels were provided in single dose tubes with applicators that were terminally sterilised. The first intravaginal dose was administered by the study physician and, provided that this was well tolerated, subsequent applications were inserted daily around the same time by the volunteers themselves. On the first day (day 0), Belgian volunteers applied one dose, UK women used two doses. On subsequent days, single doses were applied until a cumulative total of 14 doses was administered.

SUBJECT POPULATION

Subjects recruited into the study provided written informed consent. Inclusion criteria were age between 18 and 45 years, not pregnant or lactating, willing to abstain from sexual intercourse during gel use, a normal menstrual history, and a low risk for HIV infection (as determined by interview). Exclusion criteria were known HIV infection, a current STD or genital lesion, a history of post-coital bleeding, abnormal chemistry or coagulation results (because PRO 2000 is an anticoagulant), allergy to heparin or sulphated polysaccharides, the use (within 30 days of enrolment) of anticoagulants, medications with antiplatelet activity, or drugs which are cleared through the reticuloendothelial system, and the use of intravaginal products other than the study gel. At screening the investigators assessed the potential subjects' menstrual cycle to ensure that menstruation was not expected to occur during gel use.

\section{SUBJECT EVALUATION}

There were six scheduled study evaluations: screening (day -14), baseline (day 0), 2 hours after the first gel dose (day $0+2$ hours), 1 week (day 6), and 2 weeks (day 13) after starting the study product and 1 week after discontinuing the study gel (day 20). All study subjects at a given site were evaluated by the same study physician in order to eliminate interobserver error. Symptoms of vulval or vaginal soreness or pruritus, vaginal bleeding, or dysuria were recorded by participants in a diary. The potential for systemic absorption of the active agent was assessed by measuring plasma levels of
PRO 2000 and by performing laboratory safety tests, including coagulation tests at days -14 , $0,6,13$, and 20. Empty and unused applicators were returned to the trial centres as a measure of protocol adherence.

COLPOSCOPY

Macroscopic evidence of genital mucosal changes was obtained using a colposcope and findings were recorded according to modified WHO criteria (that is, no use of acetic acid, iodine or green filter). ${ }^{16}$ At the UK site, colposcopic images were stored on a computer to allow for independent evaluation at a later date.

\section{HISTOLOGY}

Microscopic evidence of vaginal inflammation was obtained using high vaginal biopsy specimens taken during the proliferative phase of the menstrual cycle (day -14 and day 13) from predetermined sites, using methodology previously reported. ${ }^{8}$ Histology was carried out by a single observer (LP) blinded both to the gel used and to whether the specimen was taken before or after exposure. Where evidence of an inflammatory infiltrate was seen, specimens were further assessed using the immunohistological technique previously described. ${ }^{6}$ The inflammation score used was: $0=$ none, no inflammatory cells; $1=$ mild, single small focus of inflammatory cells; $2=$ moderate, several foci of inflammatory cells; $3=$ severe, overt inflammation. Vaginal biopsy was carried out in the UK cohort only.

\section{MICROBIOLOGY}

An STD screen was carried out at each visit using methods previously described. ${ }^{8}$ High vaginal swabs taken at each visit were cultured for a quantitative estimation of vaginal lactobacilli as described previously. ${ }^{8}$ Vaginal flora were sampled at 12 hours after the last gel dose during the exposure phase of the study in the UK cohort whereas in the Belgian cohort, sampling was carried out 2 hours after gel insertion.

\section{SAMPLE SIZE AND ANALYSIS}

Assuming an underlying adverse event frequency of $20 \%$ in any given arm, 24 participants per group would provide a $99.5 \%$ chance of observing at least one adverse event in a given group. If the underlying frequency was $10 \%$, this chance would be $92 \%$.

For categorical variables, the number and percentage of each category within a variable was calculated. For continuous variables the mean, median, standard deviation, as well as the minimum and maximum values were determined. Owing to the modest sample size within each intervention arm, inferential assessment to compare the treatment groups was not performed. All subjects were included in the analysis.

\section{Results}

ENROLMENT

A total of 73 participants was recruited across the two study sites. In Antwerp 37 women were enrolled between January and March 1997 and in London 36 participants were recruited 
between March and September 1997. Twenty four women were randomised to each of the active groups and 25 to the placebo arm of whom 20, 24, and 25 women completed the study in the $4 \%, 0.5 \%$, and placebo groups respectively. Of the 24 participants enrolled in the $4 \%$ group, three discontinued because of adverse events (described below). The mean age was similar in all groups: 30.2 years, 33.2 years and 31.8 years in the $4 \%, 0.5 \%$ and placebo groups respectively. Compliance with use of the study gel was very high. Three women missed one dose and one missed two doses. Four women had sexual intercourse while using the gel, but continued as per protocol and were followed up accordingly.

\section{WITHDRAWALS}

Three participants from the $4 \%$ group withdrew because of adverse events. London participant 09/09 discontinued on day 6 after administering six doses (two doses on day 0 and one each on days 1, 2, 3, and 4). Moderate vulvar irritation (burning and soreness) was reported on days 0 and 1 , and mild vulvovaginal irritation on day 2. No irritation was reported from day 3 onwards, even though dosing had not yet been discontinued. Intermenstrual bleeding began on day 5 and continued until day 11. At a day 6 clinic visit, vaginal bleeding and cervical erythema were observed. Intermenstrual bleeding stopped a few days before the next visit on day 13. Bleeding attributable to normal menses began on day 13, but other signs and symptoms had resolved by that date.

Antwerp participant 01/02 discontinued dosing on day 4 after applying daily doses on days $0-3$. Mild to moderate vaginal soreness and gastrointestinal symptoms (cramps, nausea, diarrhoea) were reported during the dosing period. Small vulvar ulcers, vaginal erythema, and abrasion were observed at the day 4 visit (see colposcopic findings below). All treatment emergent findings had resolved by the next clinic visit on day 7 .

Antwerp participant 08/07 experienced abdominal pain and diarrhoea after applying the day 0 dose. All symptoms had resolved by the next visit on day 6. Apart from a cervical ectropion, which was observed throughout, colposcopic findings were unremarkable.

COLPOSCOPIC FINDINGS

In both sites colposcopy revealed few cases of epithelial disruption (table 1). Vulvar ulceration was seen on day 4 in one subject who had discontinued dosing on day 3. Clinically the appearance was suggestive of herpes simplex virus (HSV) infection, but the ulcers were not tested for HSV. Cervicovaginal ulcer was observed in four participants (one $4 \%$ and three $0.5 \%$ dose groups), all at a post-dosing visit. Two of these findings (one $4 \%$ and one $0.5 \%$ dose groups) were attributable to a healing biopsy site, and none was considered to be probably or definitely related to PRO 2000 gel.

Genital abrasion was observed infrequently in all dose groups (table 1). Three participants (one in each dose group) reported having
Table 1 Combined colposcopy results

Number of participants (*) with finding

$\begin{array}{lllll}\text { Assessment } & \text { Predose Week } 1 & \text { Week } 2 & \text { Postdose }\end{array}$

\section{Cervicovaginal ulcer:}

$4 \%$ PRO 2000 gel

$0.5 \%$ PRO 2000 gel 0

Placebo gel

0

Cervicovaginal abrasion:

$4 \%$ PRO 2000 gel

$0.5 \%$ PRO 2000 gel 1

Placebo gel

Subepithelial haemorrhaget:

4\% PRO 2000 gel 1

$0.5 \%$ PRO 2000 gel 0

Placebo gel

Erythema/oedema:

$4 \%$ PRO 2000 gel

$0.5 \%$ PRO 2000 gel 0

Placebo gel

$\begin{array}{lll}0 \ddagger & 0 & 1 \\ 0 & 0 & 3 \\ 0 & 0 & 0 \\ 3 & 2 & 2 \\ 1 & 0 & 0 \\ 0 & 1 & 2 \\ & & \\ 6 & 6 & 3 \\ 2 & 4 & 3 \\ 3 & 2 & 2 \\ & & \\ 10 & 4 & 1 \\ 5 & 5 & 0 \\ 2 & 4 & 2\end{array}$

${ }^{\star} \mathrm{N}=24$ in the treatment groups; $\mathrm{N}=25$ in the placebo arm. †Ecchymosis, petechial haemorrhage, and/or subepithelial haemorrhage with swelling.

$\ddagger$ In Antwerp a vulvar ulceration was diagnosed on day 4 for participant $01 / 02$.

sexual intercourse during the dosing period, and this may have caused the abrasions observed at the week 1 and week 2 visits in these individuals. In two others $(4 \%$ dose group), the investigator attributed the finding to applicator/instrument use. In four cases (two $4 \%$, two $0.5 \%$ dose groups), abrasions resolved during continued dosing.

In both centres, colposcopy revealed nonulcerative findings (subepithelial haemorrhage, erythema, oedema) in a minority of participants across all the dose groups (table 1).

\section{HISTOLOGY}

At the UK site, vaginal biopsies were examined microscopically after haematoxylin and eosin staining for the presence of inflammatory cells. Foci of inflammatory cells were seen microscopically in $5 / 36$ samples collected before exposure (two $4.0 \%$, two $0.5 \%$, one placebo dose groups) and in 5/35 post-exposure (two $4.0 \%$, three placebo). In one subject $(0.5 \%$ group) the post-exposure sample was not suitable for analysis (table 2). The observed inflammation did not correlate with colposcopic findings or reported symptoms.

LABORATORY SAFETY SCREENS AND SYSTEMIC ABSORPTION

No clinically significant treatment emergent changes were observed in physical examination

Table 2 Summary of biopsy scores taken at screening and day 13 at the London site

\begin{tabular}{|c|c|c|c|c|}
\hline & & $\begin{array}{l}4 \% \text { PRO } \\
2000 \mathrm{gel}\end{array}$ & $\begin{array}{l}0.5 \% \mathrm{PRO} \\
2000 \mathrm{gel}\end{array}$ & Placebo gel \\
\hline \multicolumn{5}{|l|}{ No change: } \\
\hline Screen & Day 13 & Number & Number & Number \\
\hline 0 & 0 & 9 & 9 & 9 \\
\hline 2 & 2 & 0 & 0 & 1 \\
\hline \multicolumn{5}{|l|}{ Increase: } \\
\hline Screen & Day 13 & Number & Number & Number \\
\hline 0 & 1 & 1 & 0 & 2 \\
\hline 1 & 2 & 1 & 0 & 0 \\
\hline \multicolumn{5}{|l|}{ Decrease: } \\
\hline Screen & Day 13 & Number & Number & Number \\
\hline 1 & 0 & 1 & 2 & 0 \\
\hline
\end{tabular}

Inflammation score :

$0=$ None, no inflammatory cells;

$1=$ Mild, single small focus of inflammatory cells;

2 = Moderate, several foci of inflammatory cells;

$3=$ Severe, overt inflammation. 
or laboratory factors (serum chemistry, haematology, coagulation time, urinalysis). PRO 2000 was undetectable in plasma samples collected from all participants using a validated high performance liquid chromatography method (40 $\mathrm{ng} / \mathrm{ml}$ limit of detection).

\section{VAGINAL MICROENVIRONMENT}

Very few participants had genital infections at baseline in either the London or Antwerp cohort. There was evidence of non-specific cervicitis in two women (placebo group), vaginal candidiasis in three subjects (one in each dosing group) and cervical chlamydial infection in one subject (placebo group). Vaginal $\mathrm{pH}$ was within the normal range in each of the study arms.

In the London centre, in which samples for lactobacilli assessment were collected approximately 12 hours after gel application, treatment had no effect on vaginal lactobacilli populations. Twenty eight women were assessed for changes in vaginal flora by culturing samples taken at days $0,6,13$ (12 hours after the last gel application), and 20. Four women had no lactobacilli throughout the study period. An additional four had no lactobacilli growth on day 0 . For 20 women vaginal lactobacilli levels were consistent with a normal healthy vaginal flora ${ }^{17}$ throughout the study period.

In Antwerp, a reduction in lactobacilli numbers was observed in samples collected 2 hours after the last gel application (all dose groups). In the $4 \%, 0.5 \%$, and placebo group three, five, and five women respectively had very few lactobacilli on day 14 whereas at screening they were numerous. In both active groups six women with a positive lactobacilli culture at screening had no growth on day 14 . This was also observed in five women in the placebo arm. For all participants lactobacilli populations returned to baseline levels 7 days post-dosing.

\section{SYMPTOMS AND ACCEPTABILITY}

Vaginal symptoms were reported by four, six, and nine women in the $0 \%, 0.5 \%$, and $4 \%$ group respectively. More women reported vulval irritation $-11,9$, and 10 respectively. There was no obvious correlation between these reports and observed colposcopic findings or microscopic evidence of inflammation.

Vaginal discharge attributable to gel expulsion was reported by the majority of participants in both sites $(72 \%, 92 \%$, and $83 \%$ in the placebo, $0.5 \%$, and $4 \%$ group respectively), and was observed during gynaecological examination. Normal cervical discharge was observed in about a third of the participants in each dose group before, during, and after dosing. Most subjects found the consistency, colour, and odour of the gels acceptable ("liked a lot" or "liked somewhat"), and more than half of the respondents never experienced problems with gel expulsion/leaking.

\section{Discussion}

The results of this phase I clinical trial indicate that repeated intravaginal application of gels containing up to $4 \%$ PRO 2000 was safe both locally and systemically, had no measurable impact on vaginal lactobacilli, and was well tolerated in healthy, sexually abstinent women. Though firm conclusions regarding relative toxicities are difficult to draw from these small observational studies, there was the suggestion that the 4\% PRO 2000 gel was probably approaching the dose limit for tolerance. Colposcopy showed lesions without an epithelial disruption. Their relevance to HIV is unclear. Similar findings have been seen in other studies with intravaginal products ${ }^{18}$ and in women not using vaginal substances. The reduction in lactobacilli observed in Antwerp may have been due to a sampling artefact because samples were taken immediately after the last gel application. In London samples were taken after 12 hours only; there, no effect on lactobacilli was observed. The investigators concluded that additional clinical evaluation of PRO 2000 gel is warranted, and phase II studies are planned.

We acknowledge MRC, UK and Yvette Jacob.

Contributors: LVD was responsible for the protocol, study supervision, and was the main author; $\mathrm{KD}$ was the study clinician in Antwerp and made comments on the article; VV was the statistician; EVD is head of the laboratory in Antwerp and commented on the article; ML was responsible for the protocol, supervision, and commented on the article; JW was responsible for the protocol, supervision, and commented on the article; VK

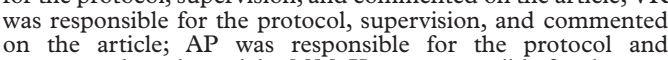
on the article; $\mathrm{AP}$ was responsible for the protocol and
commented on the article; $\mathrm{MMcK}$ was responsible for the protocol, monitoring, and commented on article; AW was the histologist; IR was the microbiologist; LP was responsible for the tologist; IR was the microbiologist; LP was r
histopathology done in London for the study.

1 Elias CJ, Heise LL. Challenges for the development of female-controlled vaginal microbicides. AIDS 1994;8: $1-9$.

2 Global Programme on AIDS, World Health Organisation. Report on a meeting on the development of vaginal microbicides for the prevention of heterosexual transmission of HIV. Geneva: GPA/WHO 1993. (Document WHO/GPA RID/CRD/94.1:11-13 November 1993.)

3 Hicks DR, Martin LS, Getchell JP, et al. Inactivation of HTLV-III/LAV-infected cultures of normal human lymphocytes by nonoxynol-9 in vitro. Lancet 1985;2:1422 3 .

4 Harrison C, Chantler E. The effect of nonoxynol-9 and chlorhexidine on HIV and sperm in vitro. Int $\mathcal{F}$ STD AIDS 1998;9:92-7.

5 Roddy RE, Zekeng L, Ryan KA, et al. A controlled trial of nonoxynol-9 film to reduce male-to-female transmission of sexually transmitted diseases. N Engl f Med 1998;339:50410 .

6 Stafford MK, Ward H, Flannagan A, et al. Safety study of nonoxynol-9 as a vaginal mircobicide: evidence of adverse effects. I Acquir Immune Defic Syndr Hum Retroviro 1998;17:327-31

7 Rosenstein IJ, Stafford MK, Kitchen VS, et al. Effect on normal vaginal flora of three intravaginal microbicidal agents potentially active against human immunodeficiency virus type 1. F Infect Dis 1998;177:1386-90.

8 Rosenstein IJ, Fontaine EA, Morgan D, et al. Relationship between hydrogen peroxide-producing strains of lactobacilli and vaginosis-associated bacterial species in pregnan women. Eur 7 Clin Microbiol Infect Dis 1997;16:517-22.

9 Balzarini J, Naesens L, Verbeken E, et al. Preclinical studies on thiocarboxanilide UC-781 as a virucidal agent. AIDS 1998;12:1129-38.

10 Ongradi J, Ceccherini-Nelli L, Pistello M, et al. Acid sensitivity of cell-free and cell-associated HIV-1: clinical implications. AIDS Res Hum Retroviruses 1990;6:1433-6.

11 Klebanoff SJ, Coombs RW. Virucidal effect of Lactobacillus acidophilus on human immunodeficiency virus type 1: possible role in heterosexual transmission. $f$ Exp Med 1991;174:289-92.

12 Sonderfan AJ, Chancellor T, Buckheit Jr RW, et al. Safety and in vitro efficacy of a topical microbicide gel for the prevention of HIV-1 transmission. 4th Conference on Retroviruses and Opportunistic Infections, Washington DC, January 1997 (Abstract 522).

13 Profy AT, Sonderfan AJ, Bernstein DI, et al. PRO 2000 gel, a potential topical microbicide for HIV prevention, can block infection by other sexually transmitted disease pathogens. 12th World AIDS Conference, Geneva, June pathogens. 12th (Abstract 33146)

14 Bourne N, Bernstein DI, Ireland J, et al. The topical microbicide PRO 2000 protects against genital herpes infection in a mouse model. F Infect Dis (in press).

15 Sonderfan AJ, Chancellor T, Buckheit RW Jr, et al. Safety and in vitro efficacy of a topical microbicide gel for the pre- 
vention of HIV-1 transmission. 4th Conference on Retroviruses and Opportunistic Infections, Washington DC, 22-26 January 1997.

$16 \mathrm{WHO}$ WHO/GPA/RID/CRD. Manual for the standardization of colposcopy for the evaluation of vaginally administered products. Geneva: World Health Organisation, 1995.
17 Hillier SL. Recolonisation of the vagina with an exogenous strain of Lactobacillus crispatus. BV 98 Aspen Colorado, 17-19 September 1998.

18 Van Damme L, Niruthisard S, Atisook R, et al. Safety evaluation of nonoxynol-9 gel in women at low risk of HIV infection. AIDS 1998;12:433-7. 